\title{
A Heritage Economy: Business Model Innovation for Economic Wealth, Social Well-Being, and Environmental Health
}

\author{
Frédérick Brousseau-Gauthier and Yvon Brousseau
}

\author{
"You don't change a company by giving them ideas." \\ You change them by training them to think a \\ different way.
}

\author{
Clayton Christensen \\ Professor of Business Administration, \\ author, and consultant
}

\begin{abstract}
Faced with the inherent unsustainability of infinite growth in a world of finite resources, the neoclassical economy is running towards a cliff. In order to avoid a hard landing, enterprises need to broaden their definitions of value and wealth to include parameters that are not currently in the economic lexicon, but are still of paramount importance in our lives. Taken from that angle, heritage can be seen as a perfect replacement for capital, because its multidimensional and complex nature opens up numerous possibilities for the creation of shared economic, social, and environmental value; the designing of value chains; and the direction of technological innovation. This article explores the various ramifications of a paradigm shift from managing capital to managing heritage, and it underlines the need to create a series of pioneering business models for enterprises to adapt and profit from a new, heritage economy.
\end{abstract}

\section{Introduction}

Today's market, as conceived by neoclassical economists, is ongoing a triple crisis that severely circumscribes the growth prospects for companies and jeopardizes their mid-term and long-term profitability. On one side, enterprises must address growing political and social pressure to act as corporate citizens, namely by taking on greater responsibility regarding the impacts of their operations on the communities living in the area where they are established, and by acknowledging that social acceptance is an essential criteria for their projects to be achieved. On another side, population growth, resource scarcity, problematic access to energy, and the deterioration of the environment by pollution create instability in the supply of raw materials, extra costs due to the need for decontamination, and productivity losses for companies. Lastly, whereas the world's population will rise above 9 billion by 2050, the increasing complexity of the global market is already problematic. In particular, marketing and financing processes have grown to a scale that most business models are unsuited for, causing multiple difficulties for the commercialization of innovations.

For enterprises, addressing this triple crisis is proving to be as much of a necessity as it is a challenge. It is a necessity because the crisis has its roots in a fundamental flaw in the neoclassical conception of the economy: whereas the sum of all human and natural resources is finite - and is therefore convergent - the market operates as if growth was to be infinite - and is therefore divergent. In the long run, this situation is obviously unsustainable. It is a challenge because, to establish and maintain their competitiveness in uncertain market conditions, multinationals and small- and medium- 


\section{A Heritage Economy}

Frédérick Brousseau-Gauthier and Yvon Brousseau

sized enterprises alike must re-examine their relationship with wealth and reconcile their operations with the real economic space that is available to them. Companies must broaden the parameters of corporate decision making to exceed the scope of capital management and adopt a paradigm more suited to the analysis of multiple interdependent parameters. In this article, we argue that this paradigm centers on "heritage".

\section{A Heritage Economy}

Heritage can be defined as an intertwinement of being and having that derives its sense and value from continuous relationships between it and a human community (Vivien, 2009; tinyurl.com/nj4jvqu). It is therefore a set of essentially complex elements, whose worth is embedded in a specific culture and thus cannot be entirely marketed. Heritage can be tangible - like water, locations, buildings, etc. - or intangible - like knowledge, art, and energy. However, given that discussions on the heritage-management approach have been centered so far on heritage objects, this article will concentrate on the tangible forms of heritage.

Thus, for example, a fresh water source is at the same time providing natural services and helping to define a community's identity; as the settling of a group of humans near a river or a lake helped to shape this group's history and culture over time, the geographical feature became a part of the community's heritage.

It follows that managing a fresh water source strictly as a provider of natural services that can be monetized and therefore sold to the highest bidder - is both disrespectful of its heritage nature and cannot ensure that its use is going to be in agreement with the long-term interests of the community. Therefore, companies that wish to access a specific heritage element need to move away from the maximization of short-term profits derived from its exploitation and refocus on the creation of a sustainable appropriation structure that guarantees its long-term preservation and florescence - in the sense that heritage is to be seen as a vital organism that grows in the social ecosystem that is a community. This change of focus will enable firms choosing a heritagebased approach to establish themselves as channels of economic wealth, social well-being, and environmental health for the community whose valued heritage is used for commercial purposes, thereby creating a context of stability and durability for businesses to thrive in. Thus, to fully enroll in the new heritage-management paradigm, companies must broaden the paramet- ers of corporate decision making to include what they consider to be externalities, but truly are effects of their appropriation of the natural, human, and financial resources that sustain them. Henceforth, the social and environmental impacts of their operations must be given the same weight as their economic impacts. The adoption of this global approach alters the cost-benefit analysis parameters to the point that it necessitates the inclusion of all individuals, groups, and other stakeholders that are affected by this appropriation of a part of their heritage.

The appropriation process for a heritage object, location, work of art, and so forth establishes a relationship that goes beyond the concept of property. It involves, for example, putting forward: i) a specific portrayal of a heritage object (e.g., how the company views it, namely as a revenue source); a precise use for this object (e.g., its exploitation for business ends); policies for access and the transfer of access rights; and an allotment structure for revenues derived from this appropriation (e.g., profits distributed among the shareholders, taxes, and other dues paid to the state) (Weber and Reveret, 1993; tinyurl.com/ozolqdq).

Although from a strict capital-management standpoint being the owner of the access rights to an object gives companies full discretion to maximize their profits, heritage management requires them to take all facets of the appropriation process into account. Indeed, the heritage nature of said object requires enterprises that hold rights to it to establish a decision-making process that considers the multiple representations and uses that this object already has, and that are valued by the community that has had a continuous relationship with this part of their heritage (Weber and Reveret, 1993: tinyurl.com/ozolqdq; Vivien, 2009: tinyurl.com/nj4jvqu). The acknowledgement of this non-marketable value must fuel negotiations that will in turn lead to the recognition of all representations associated with the heritage object - including those of the enterprises - in order to establish the architecture of the appropriation process. These negotiations, together with an evolving decisionmaking process - as opposed to a static and conclusive one - will allow the founding of a heritage-appropriation structure that will ensure the economic, social, and environmental profitability of its use, and thus its social acceptability.

However, seeking an optimal state in the management of heritage will require companies to reach a new level of efficiency in the setup of their operations in order to 


\section{A Heritage Economy}

\section{Frédérick Brousseau-Gauthier and Yvon Brousseau}

reach that objective. This task will be highly complex because the internalization of externalities will force organizations to restructure all facets of their business from their core structure to how decisions are made (Willard, 2013; tinyurl.com/lw6m67q). Hence, it will be necessary to create new business models that take those externalities into account in the design of value chains the structure underlining production, distribution, sale, and profit. In this context, firms that create business models that integrate, promote, and distribute shared economic, social, and environmental value will be best equipped to address these new market conditions and will achieve strategic advantage, in the same way that developing a new technology provides such an advantage.

\section{Business Model Innovation}

Business models articulate the logic, the data, and other evidence that support a value proposition for the customer, and a viable structure to manage revenues and costs for the company delivering that value in order to make profit (Teece, 2010; tinyurl.com/oduv9wl). Enabling greater productivity, efficiency, and inimitability are the positive attributes that raise a business model to the status of strategic and competitive advantage for an enterprise. The heritage economy explicitly entails the long-term preservation and florescence of heritage rather than its short-term exploitation, the transcending of property as the sole justification for appropriation, together with the broadening of the decision-making power to include stakeholders and the changing of its process (Vivien, 2009; tinyurl.com/nj4jvqu). Therefore, 21st century business models need to include the following three major notions:

1. Revenue interdependency is a state of symbiosis between companies, customers, and heritage that involves a shift from the exploitation of both heritage's natural services and customers' paying ability, to the creation of shared value. When a company appropriates a heritage object for business purposes, it creates a relationship where the company benefits from the preservation of said heritage object because: i) it makes possible the continuous harvesting of value from it; ii) it ensures the livelihood of the community to which the company sells its value propositions; and iii) it secures long-term social acceptability for the company's operations. From a heritage standpoint, a company that creates economic wealth, social well-being, and environmental health from its appropriation of a heritage object - through both sustainable harvesting and the nature of its value propositions - contributes to an increase in the worth of the object and therefore creates shared value for the community. Thus, awareness of the interdependency between companies, customers, and heritage (as seen in Figure 1) urges the former to develop business practices that are beneficial for all stakeholders: practices that create shared value, whose growth then enables the long-term florescence of heritage.

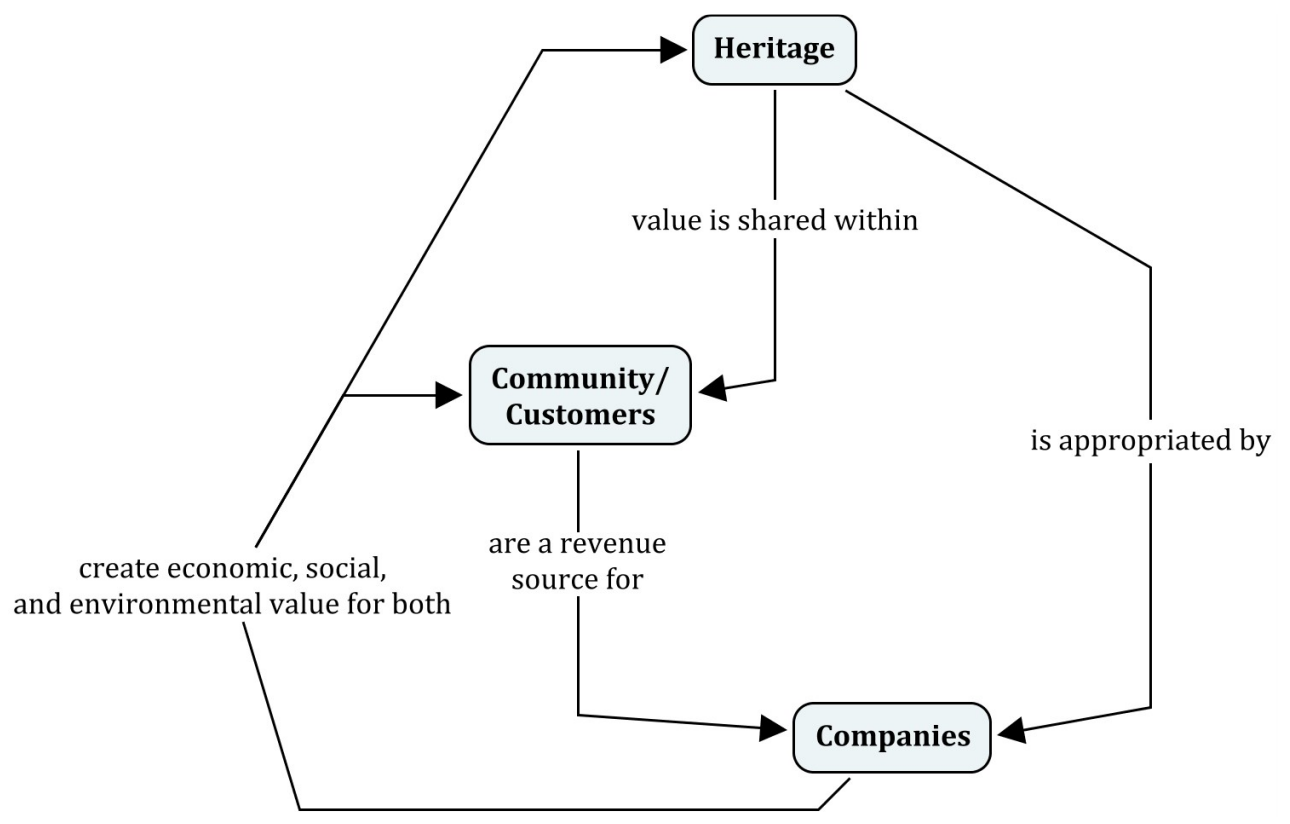

Figure 1. The interdependency between companies, customers, and heritage 


\section{A Heritage Economy}

\section{Frédérick Brousseau-Gauthier and Yvon Brousseau}

2. Free access removes limitations on access to all goods, services, and technologies created by humans to allow the greatest possible circulation of their benefits across society. However, free access is not free use, quite the contrary; it leads to a change to the way corporations capture value, from benefitting from selling property or an access to property to benefitting from selling the use of goods or services. Thus, free access naturally thrives in the context of a shift from the selling of goods to the provision of services. Free access also moves the setting of wealth creation outside the scope of property to place it in the perspective of heritage management, which further upholds the importance of stakeholder involvement in the decision-making process (Weber and Reveret, 1993; tinyurl.com/ozolqdq).

3. Knowledge sharing refers to the opening of data and the increased exchange of information. This opening will be made necessary by the level of complexity required for enterprises to maximize the creation of economic wealth, social well-being, and environmental health. To achieve this objective in an optimal way, companies must adopt policies of acute transparency regarding their operations and business practices. It is only the automatic sharing of data that will enable all stakeholders to gather the knowledge required to design suitable solutions for companies, so the latter can successfully address the complex issues of heritage management and gain public acceptance.

It will take ample research and creativity to embed these three notions into highly effective business models that address the needs of companies. This need has brought forth the idea of the Hub for Business Model Innovation (Hub-BMI), a research centre that will enable the development, testing, and validation of pioneering business models. Founded by the Centre of Excellence in Energy Efficiency (C3E; c3e.ca), which is the architect of the project, the Hub-BMI is based in Montréal and is now at the development and fund-raising stage. It has already started to draw on the expertise of highly skilled professionals who hail from a large network of multinationals, small- and medium-sized businesses, governments, cities, and financial and academic institutions. An interdisciplinary approach that brings together economic, artistic, and intellectual ecosystems will allow the Hub-BMI to gather the creative power needed to design quality tools that will enable enterprises to adapt to changing market conditions.
For example, although promising, the transition from capital management to heritage management is constrained by an outdated legal framework that is not equipped to accommodate for-profit entities whose social and environmental benefits purposes are central to their existence (Clark and Vranka, 2013; tinyurl.com/ lampp6b). The establishment of an economy based upon heritage management therefore requires a new type of corporate legal entity - the benefit corporation (Clark and Vranka, 2013; tinyurl.com/lampp6b) - that will in turn enable the internalization of social and environmental externalities in the decision-making process. As a result, benefit corporations that join in the heritage economy will distinguish themselves as leaders of this new paradigm and achieve strategic positioning in their field by establishing enduring relationships with stakeholders of their economic, environmental, and social ecosystem. Furthermore, the adoption of a heritagemanagement approach - as opposed to a sole focus on capital management - will facilitate compliance with impact investment standards (Willard, 2013; tinyurl.com/ lw6m67q) and will contribute greatly to the singling out of these enterprises by investors looking for such opportunities (Unsworth, 2012: tinyurl.com/n9mhtf5; Canadian Task Force on Social Finance, 2011: tinyurl.com/phmmx6s).

\section{Natural Capitalism}

The goal we hope to achieve by moving to a heritage economy is to close the gap between economic activity and the activities of humans and ecosystems. In itself, it means reconciling capitalism with the human, social, and environmental planet on which it evolves, to naturalize it in a way that the market integrates the whole instead of distancing itself from it, severed from the complex and multidimensional reality of the world. This naturalization - based upon a threefold approach that measures the economic, social, and environmental impacts of using heritage to business ends - should produce two kinds of major repercussions in how economic activities are designed and managed: i) it will aim technological innovation towards a greater integration with the environment and ii) it will reorganize the structure of value chains in order to eliminate all forms of muda (Hawken et al., 1999; tinyurl.com/lzzxegy).

Muda is Japanese for "waste", "futility", or "purposelessness". Within the context of economics, any human activity that absorbs resources but creates no value classifies as muda (Hawken et al., 1999; tinyurl.com/lzzxegy). Understandably desirable, muda's systematic elimina- 


\section{A Heritage Economy}

\section{Frédérick Brousseau-Gauthier and Yvon Brousseau}

tion was theorized then applied to industrial manufacturing processes and framework by Taiichi Ohno (tinyurl.com/mkykkra). Within such an optimized system, the value chain is designed to be a continuous flow of value, as defined by the customer, at the pull of the customer, and in search of perfection - which is in the end the elimination of muda (Womack and Jones, 2003; tinyurl.com/kw9v74h).

There are many different ways that companies can optimize their value chains in order to eliminate muda, leading to increased profitability. The following concepts, presented as complementary strategic axes in the book Natural Capitalism (Hawken et al., 1999; tinyurl.com/lzzxegy), represent some of the most promising approaches:

1. The first axis is the radical improvement of natural and human resource productivity. Given that, within this context, the term "productivity" refers to the amount of output a process provides by unit of input, increased resource productivity means obtaining the same amount of utility or work from a product or process while using less material and energy (Hawken et al., 1999; tinyurl.com/lzzxegy). Just as the industrial revolution allowed a phenomenal increase in the productivity of workers, which resulted in the expansion of production by the means of energy access, mechanization, the assembly line, and so on, a revolution in resource productivity would allow the intensification of production and would have numerous positive impacts. For example, the reorganization of value chains and the integrated use of new technologies that use less energy or raw materials could slow resource depletion upstream, reduce pollution downstream, and increase the profitability of enterprises, thereby creating incentives for job creation. Given that an alternative to traditional industrial development is needed to provide growth possibilities to an increasing global population, specifically in the case of the emerging markets, revolutionary leaps in resource productivity will provide an opportunity for corporations to save money, as well as a sustainable way to increase the quality of life of people around the world. Examples of technologies that could help to achieve such revolutionary leaps such as zero-energy buildings, super-refrigerators, biointensive minifarming, perennial polyculture, etc. - are elaborated in Factor Four: Doubling Wealth, Halving Resource Use: The New Report to the Club of Rome (Weizsäcker et al., 1998; tinyurl.com/m69yd9s).
2. The second axis is biomimicry in the design of production processes and flow of materials. Indeed, biological systems naturally tend to optimize the use of inputs in order to minimize waste, because, by contrast with mechanical systems, they are not artificially maintained by a constant supply of resources and power. Biological assembly techniques are thus optimized to occur in low-temperature, low-pressure environments, and to require minimal energy. Therefore, replicating nature's assembly techniques opens the door to reaching extraordinary levels of resource productivity. The goal of biomimicry ultimately is to structure the production processes so that they imitate biological cycles, which work in closed cycles where every species' waste is another's sustenance. Given that, in economic terms, waste is a loss of capital, its elimination - by the company finding another use for the waste within its operations, by selling the waste to another firm which has a use for it, or by other means - constitutes a new appreciation of this capital as well as a revenue source for enterprises.

3. The third axis is a fundamental shift from the selling of goods to the provision of services in the customerproducer relationship. This change is based on the idea that customers want solutions to their real and perceived needs and not the possibility of owning goods that are supposed to meet their requirements. For enterprises, this means moving to the licensing of goods of which they will effectively retain the ownership, and, consequently, be responsible for their maintenance. The main effect of this change will be an alignment of interests by enterprises and customers as to the durability of the object providing services: by contrast with a ownership approach where it is in the interest of companies to design products that require frequent replacement, enabling them to repeatedly sell the same product to a group of customers - an approach based on the provision of services drives enterprises to design their products so that they will be as durable as possible. In the same line of thought, choosing to provide services instead of selling goods provides enterprises further incentives to improve their resource productivity and to implement biomimetic production processes, thereby reducing the cost of product maintenance and protecting their investment.

The integration of these three strategic axes in the architecture of value chains will form the cornerstone of a new generation of business models. These new busi- 


\section{A Heritage Economy}

Frédérick Brousseau-Gauthier and Yvon Brousseau

ness models will in turn define strategies of distribution, highlighting, and profitability that will support the commercialization process of technological innovations. The heritage approach, and the new paradigm that will follow its spread, will provide incentives for companies to become benefit corporations: the type of firms that are destined to become the primary focus of impact investors (Unsworth, 2012: tinyurl.com/n9mhtf5; Canadian Task Force on Social Finance, 2011: tinyurl.com/phmmx6s).

\section{Conclusion}

In a world of increasing interconnection, enterprises' responsibility in the management and preservation of natural, human, physical, and cultural heritage will continue to grow with the capacity of enterprises to affect the condition of this heritage. The need to broaden economic considerations to a larger scope, that of the heritage economy, will only be made more obvious by the increasingly spectacular impacts of economic activity on social well-being and environmental health.

Although the awareness of this necessity spread across business and political circles, the creation of the HubBMI introduced above will institute a gathering of creative power capable of tackling the first step towards a heritage economy: the design of a series of business models that will act as instruction manuals for the conversion of enterprises to this new economy.

Nevertheless, it will be quite a challenge for humanity to move to a heritage economy. Beyond fundamental and applied research, it will take a paradigm shift that will transform our elementary notions about commerce to successfully face the neoclassical economy's triple crisis. In that regard, the state of the world today acts as a powerful incentive to achieving this new Renaissance, so as to reconcile economic activity with the preservation and florescence of heritage. Above all, it will take ample courage and determination to stir the rise of humanity as a growing and stability-inducing species on this planet.

\section{About the Authors}

Frédérick Brousseau-Gauthier is a creative writing student at Université du Québec à Montréal (UQÀM), with a strong interest in the relationships between politics, the economy, and the environment. Between 2006 and 2008, he took part as speaker in several seminars in France and Canada on the topic of information technologies and their role in education. He also worked as columnist and investigative reporter in various student media outlets, covering matters from citizens' engagement in their democracy to the management of collective property. He is currently writing a novel.

Yvon Brousseau is CEO of the Centre of Excellence in Energy Efficiency (C3E), which has a mandate to develop "commercialization exit strategies" for R\&D projects from the private and public sectors across Canada. He holds a Master's degree in Business Administration and a Bachelor of Science degree in Microbiology from Sherbrooke University, Canada. He has more than 20 years of experience including his current role of CEO of C3E, during 8 years as CEO for an applied mathematical start-up company, and during 12 years as a marketing/finance consultant. He managed and operated business corporate strategies and $R \& D$ projects for different business sectors (e.g., biotech, chemistry, optics, and mathematics) with well-educated and seasoned employees from scientific, technical, and financial backgrounds.

Citation: Brousseau-Gauthier, F. and Y. Brousseau. 2013.

A Heritage Economy: Business Model Innovation for

(cc) BY

Economic Wealth, Social Well-Being, and Environmental

Health. Technology Innovation Management Review.

September 2013: 32-37.

Keywords: heritage economy, business model innovation, natural capitalism, impact investment, benefit corporation 